\title{
High-intensity interval training ameliorates high-fat diet- induced elevation of aminotransferases in male Wistar rats
}

\author{
Qazaleh Asqari $^{1}$, Farhad Gholami ${ }^{*}{ }^{\mathbb{D}}$, Jabbar Bashiri ${ }^{\circledR}{ }^{\mathbb{D}}$, Adel Donyaei $^{1}$ \\ 'Department of Sports Physiology, Faculty of Sport Sciences, Shahrood University of Technology, Shahrood, Iran \\ ${ }^{2}$ Department of Sport Sciences, Faculty of Education and Human Sciences, Tabriz Branch, Islamic Azad University, Tabriz, Iran
}

*Corresponding Author: Farhad Gholami, Department of Sports Physiology, Faculty of Sport Sciences, Shahrood University of Technology, Shahrood, Iran. Tel: +989365827050, Email: gholami-fa@shahroodut.ac.ir

\begin{abstract}
Background and aims: A high-fat diet increases triglyceride (TG) accumulations in hepatocytes and results in non-alcoholic fatty liver diseases (NAFLDs). In this regard, this study investigated the effect of high-intensity interval training (HIIT), along with a high-fat diet on the serum levels of aminotransferases in male Wistar rats.

Methods: Forty male Wistar rats were randomly assigned to the standard diet, high-fat diet, exercise + standard diet, and exercise + high-fat diet groups (each containing 10 animals). HIIT program consisted of 6-12 repetitions of 2-minute highs-intensity exercise (85-90\% of the maximum speed) interspersed with 1-minute low-intensity exercise (45-50\% peak speed) with the frequency of 5 sessions a week over 12 weeks. High-fat diet groups received a diet regimen including $58 \%$ fat, $25 \%$ protein, and $17 \%$ carbohydrate, ad libitum. The blood samples were taken from the left ventricle 48 hours following the last intervention to assess TG, alanine aminotransferase (ALT), and aspartate amino-transferase (AST) concentrations. Data were analyzed using one-way ANOVA and Tukey's post-hoc tests.

Results: The findings showed the mean of ALT, AST, and TG in the high-fat diet group was significantly greater compared to the standard diet group $(P=0.001)$. Furthermore, the mean of ALT, AST, and TG in the exercise + high-fat diet group was significantly lower in comparison with the high-fat diet group $(P=0.01, P=0.017$, and $P=0.012$, respectively).

Conclusion: Although HIIT ameliorated high-fat diet-induced elevations in the serum levels of TG, ALT, and AST, they did not reach the baseline levels. Thus, it may indicate that a diet as the underlying cause of NAFLDs is more important than any other interventions such as exercise.

Keywords: High-intensity interval training, Aspartate aminotransferase, Alanine aminotransferase, High-fat diet
\end{abstract}

Received: 8 October 2020, Accepted: 6 February 2021, ePublished: 29 September 2021

\section{Introduction}

Recent lifestyle habits such as increased consumption of high-fat diets and limited physical activity are associated with obesity, hyperlipidemia, insulin resistance, and nonalcoholic fatty liver diseases (NAFLDs) $(1,2)$. NAFLD is a metabolic condition which includes a wide range of diseases from steatosis to non-alcoholic steatohepatitis (3). Studies have shown that a high-fat diet increases triglyceride (TG) accumulations in hepatocytes $(4,5)$. Carmiel-Haggai et al suggested that a high-fat diet results in the progression of NAFLD and induces oxidative stress which aggravates the disease (6). NAFLD is characterized by the accumulation of TG in hepatocytes, which is formed by the esterification of free fatty acids and glycerol (7). Liver damage due to nonalcoholic fatty liver disease is defined as a condition in which the fat content of the liver determined is greater than $5 \%(8)$. Liver diseases can also be diagnosed by the circulatory levels of aminotransferase enzymes. The serum levels of these aminotransferases indicate the condition of hepatocytes, the increase of which is associated with liver disorders and diseases. Hou et al indicated an association between a higher alanine- amino transferase level and elevated TG concentrations in NAFLD (9). Similarly, Suzuki et al found a relationship between elevated alanine aminotransferase (ALT) levels and the risk of NAFLD (10). Lifestyle modifications such as a healthy diet and increased physical activity have been recommended to prevent liver disorders.

Aerobic exercise training is the most common exercise modality that is generally suggested to improve health status (11). Exercise recommendations to experience health effects include $150 \mathrm{~m}$ exercise with moderate intensity during a week (11). Exercise training reduces visceral fat levels while improving lipid profile and insulin resistance and has the potential to improve hepatic function (11). Slentz et al reported that aerobic and resistance training decreased liver and visceral fat accumulations and ALT levels in overweight subjects (12). High-intensity interval training (HIIT) has recently received attention because it is time-efficient and increases energy expenditure to higher levels. Falcone et al reported that HIIT requires more calories compared to steady-state exercise (13). Likewise, Yoshioka et al showed that HIIT leads to lesser boy fat which may be associated

(C) 2021 The Author(s); Published by Shahrekord University of Medical Sciences. This is an open-access article distributed under the terms of the Creative Commons Attribution License (http://creativecommons.org/licenses/by/4.0), which permits unrestricted use, distribution, and reproduction in any medium, provided the original work is properly cited. 
with elevated post-exercise energy metabolism (14). Six weeks of HIIT protocol has been reported to improve lipid profile, body fat percentage, and fitness levels in overweight and obese males (15). Regarding the effects of HIIT on fatty liver diseases, Hallsworth et al indicated that the modified HIIT program reduces liver fat and alanine transaminase (ALT) and aspartate aminotransferase (AST) levels (16). It has been suggested that HIIT is superior to traditional exercise modalities with regard to improving body fat levels in obese participants (17). In contrast, Wewege et al concluded that HIIT and moderate-intensity exercise exhibit similar effectiveness on the measures of body composition (18). More recently, Kalaki-Jouybari et al suggested that HIIT is an effective intervention for improving NAFLD in diabetic rats and seems to have a greater impact in comparison to continuous exercise training (19). Although the physiological and health effects of HIIT have received attention in recent decades, research in this area is not as extensive as other exercise modalities. Thus, the present study aimed to determine whether elevations in TG and aminotransferases induced by a high-fat diet can return to baseline levels by the HIIT program. HIIT is known to elicit high energy expenditure during exercise that can remain elevated for hours postexercise. Therefore, this study focused on identifying whether incorporating exercise training with high energy expenditure has the potential to compensate for impaired liver function as a result of high-fat diet ingestion. More precisely, the study sought to investigate the effect of 12week HIIT on TG and aminotransferases levels during high-fat diet ingestion in male Wistar rats.

\section{Materials and Methods}

\section{Animals and exercise}

Forty male Wistar rats (age: 10-12 weeks) were housed in a quiet and controlled condition (temperature: $20 \pm 2^{\circ} \mathrm{C}$, humidity: $50 \pm 10 \%$ ) under a 12-hour light/dark cycle. Animals were familiarized with exercise protocols over a familiarization week (treadmill exercise with incline: $0 \%$, speed: $10-15 \mathrm{~m} / \mathrm{min}$, duration: $5-10 \mathrm{~min} / \mathrm{d}$ ). Then, the maximal speed was assessed by an incremental exhaustive exercise test on a motorized treadmill. The initial speed was $10 \mathrm{~m} / \mathrm{min}$ with a graded increase of $3 \mathrm{~m} / \mathrm{min}$ every 2 minutes until exhaustion. The animals were defined to be exhausted if they could not perform running in spite of electric shocks. After the incremental exercise test, the animals were matched based on the weights and were randomly divided into four groups (each consisting of 10 cases) including a standard diet, high-fat diet, exercise+ standard diet, and exercise + high-fat diet. HIIT program consisted of 6-12 repetitions of 2-minute high-intensity exercise (85\%-90\% of the maximum speed) interspersed with 1-minute low-intensity exercise (45\%-50\% peak speed). The exercise protocol was applied 5 sessions a week over 12 weeks. The running speed increased to the average of $5 \%$ every week and repetitions increased fortnightly throughout the experimental period.

\section{Diet}

Groups of the standard diet were fed ad libitum with the standard rat chow and water (4\% fat, $21 \%$ protein, $52 \%$ carbohydrate, and $13 \%$ fiber). The high-fat diet included $58 \%$ fat, $25 \%$ protein, and $17 \%$ carbohydrate ad libitum (20). The water intake and feed of the animals were measured during the experimental period.

\section{Measurements}

Animals were anesthetized by ketamine and xylazine (90 $\mathrm{mg} / \mathrm{kg}$ and $10 \mathrm{mg} / \mathrm{kg}$, respectively). Blood samples were collected from the left ventricle 48 hours after the last session. Then, blood samples were centrifuged at $3000 \mathrm{rpm}$ to separate serum samples to assess AST, ALT, and TG levels. ALT and AST levels were evaluated by commercial kits (Pars Azmoon, Iran) by the photometric method, and the TG level was assessed by commercial kits (Pars Azmon) using an enzymatic colorimetric assay.

\section{Statistics}

Statistical analysis was performed by SPSS software, version 25. The normal distribution of the data was determined by the Shapiro-Wilk test. Thus, a one-way ANOVA statistical test was used to determine the difference between the groups and Tukey's post-hoc test, and $P<0.05$ was considered statistically significant.

\section{Results}

Based on the results (Table 1) the mean values for all variables (ALT, AST, and TG) significantly differed between the groups $(P<0.001)$. Tukey's post hoc analysis indicated that there was a significant increase in the mean ALT, AST, and TG in the high-fat diet group (106\%, 79\%, and $150 \%$, respectively) compared to the standard diet group $(P=0.001)$. Moreover, the mean of ALT, AST, and TG in the exercise + high-fat diet group was significantly lower compared to the high-fat diet group $(P=0.01$, $P=0.017$, and $P=0.012$, respectively, Figures $1-3)$. In

Table 1. Mean \pm SD of variables between groups

\begin{tabular}{lcccc}
\hline & Standard Diet & High-fat Diet & Exercise + Standard Diet & Exercise + High-fat Diet \\
\hline TG $(\mathrm{mg} / \mathrm{dL})$ & $79.1 \pm 11.4$ & $198.3 \pm 20.5$ & $74.9 \pm 10.3$ & $177.5 \pm 21.7$ \\
ALT $(\mathrm{U} / \mathrm{L})$ & $47.4 \pm 6.2$ & $97.8 \pm 10.9$ & $44.1 \pm 6.5$ & $74.2 \pm 8.4$ \\
AST $(\mathrm{U} / \mathrm{L})$ & $76.5 \pm 8.7$ & $137.3 \pm 10.1$ & $65.8 \pm 6.7$ & $118.5 \pm 11.3$ \\
Weight $(\mathrm{g})$ & $194.0 \pm 15.6$ & $208.4 \pm 11.7$ & $192.6 \pm 14.6$ & $195.2 \pm 10.0$ \\
\hline
\end{tabular}

Note. TG, triglyceride; SD, Standard deviation; ALT, Alanine amino-transferase; AST, Aspartate amino-transferase. 


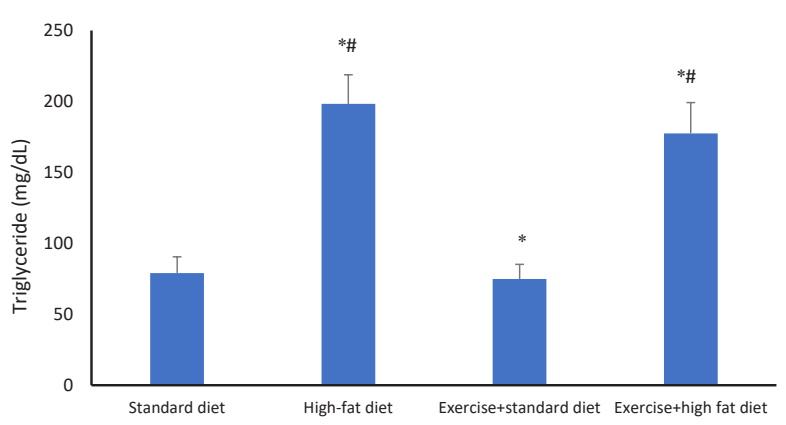

Figure 1. Triglyceride levels among groups. Note. ${ }^{*}$ Indicates a significant difference with the group receiving the standard diet $(P<0.05)$. * Represents a significant difference with exercise + standard diet $(P<0.05)$.

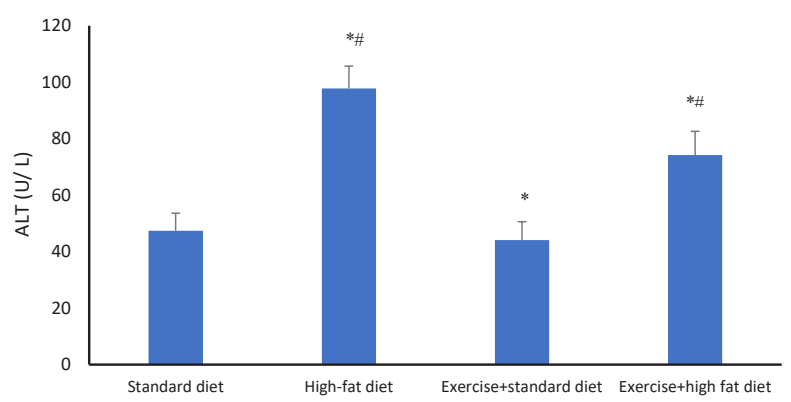

Figure 2. ALT levels among groups. Note. ALT: Alanine amino-transferase. * Demonstrates a significant difference with the group receiving the standard diet $(P<0.05)$. * Indicates a significant difference with exercise + standard diet $(P<0.05)$.

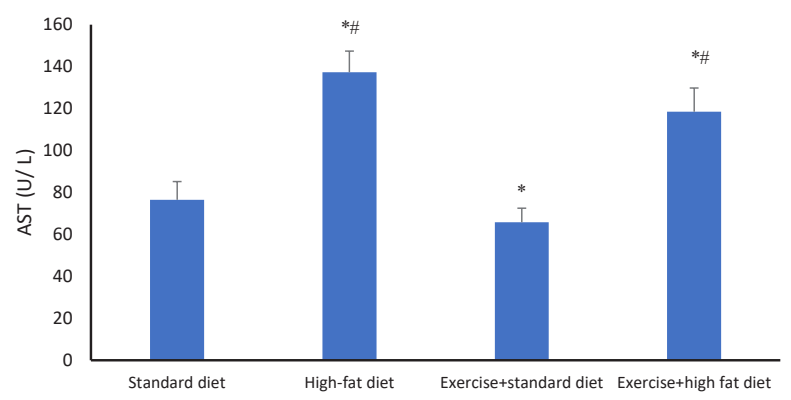

Figure 3. AST levels among groups. Note. AST: Aspartate aminotransferase. * Indicates a significant difference with the group receiving a standard diet $(P<0.05)$. ${ }^{*}$ Denotes a significant difference with exercise + standard diet $(P<0.05)$.

addition, there was a significant difference between the standard diet and exercise + high-fat diet groups, indicating that the serum levels of variables did not return to baseline values by HIIT.

\section{Discussion}

According to the main findings of the present study, a high-fat diet elevated the serum levels of TG which was accompanied by the elevation of aminotransferases. HIIT program significantly ameliorated the elevation of TG, ALT, and AST induced by a high-fat diet. However, it could not return the elevated variables to baseline levels.
As regards the effect of a high-fat diet on TG levels, our results are in line with those of Srinivasan et al, indicating that a high-fat diet significantly elevated plasma TG levels by 2 -fold in Male Sprague-Dawley rats. Elevated TG concentrations due to fat intake increase its accumulation in hepatocytes. Duarte et al reported that a high-fat diet elevated hepatic TG contents in mice, and this is due to the reestrification of the existing or ingested lipids (21). Likewise, Delgado et al indicated that hepatic TG concentrations increased about 3-fold within the first week when rats were fed with a high-fat diet, revealing the high sensitivity of hepatic TG contents to diet modifications. The components of hepatic TG may be derived from fat intake or may be synthesized within the liver by de novo lipogenesis. Similarly, Delgado et al suggested that the elevated hepatic TG concentration during a high-fat diet is almost entirely caused by dietary uptake and plasma fatty acid esterification. This shows that the contribution of de novo lipogenesis during a high-fat diet in hepatic TG is extremely little. The accumulation of TG in hepatocytes eventually leads to NAFLD (22). Dhibi et al also reported that a high-fat diet, especially the intake of trans-fatty acids, results in NAFLD (5). They further reported a significant elevation in the plasma levels of the indicators of hepatic injury, including ALT, AST, and ALP following a high-fat diet. Our results are also in conformity with the findings of Guo et al, demonstrating that a high-fat diet elevated the levels of ALT, serum cholesterol, and hepatic TG confirming that a high-fat diet induces hepatic steatosis (23). In another study, Khan et al also found that a high-fat diet led to a remarkable increase in ALT and AST in animal models (24). According to Dhibi et al, a high-fat diet causes a remarkable elevation in liver lipid peroxidation while a reduction in antioxidant defense, which can eventually, damage hepatocytes. The disrupted plasma membrane in damaged hepatocytes consequently results in the leakage of enzymes and the elevation of serum concentrations. This is manifested by a considerable increase in the circulatory levels of ALT and AST, which conforms to our results and those of the aforementioned studies.

In contrast, exercise training has been suggested to exert a protective effect on hepatocytes. It has been suggested that both continuous and accumulated exercises have the potential to reduce postprandial lipemia (25). Hao et al concluded that exercise training on the treadmill significantly reduced intra-abdominal and liver fat accumulations in ovariectomized rats (26). Regarding the effect of HIIT on the liver tissue, it has been indicated that HIIT improves insulin sensitivity while reducing fat accumulations and TG contents. Botezelli et al also reported that a fructose-rich diet decreased insulin sensitivity whereas increasing TG and AST levels in Wistar rats. They showed that exercise training successfully reduced the variables and prevented NAFLD (27). Currently, KalakiJouybari et al demonstrated that HIIT effectively improves NAFLD in diabetic rats and is more potent than continuous 
exercise training (19). Our findings corroborate with the results of the aforementioned studies, implying that the HIIT program has the potential to reduce elevated levels of the indicators of NAFLD. Several mechanisms may explain this effect of the HIIT protocol. Some studies represented that the hepatic secretion of extremely lowdensity lipoprotein triacylglycerol and postprandial triacylglycerol concentrations reduces following HIIT $(28,29)$. Furthermore, there is a link between dysglycemia and NAFLD, indicating that glucose control is of particular importance in preventing NAFLD progression. Although glucose levels were not assessed in the present study, it was found that HIIT potentially stabilizes glucose control which is practically of high importance to NAFLD (30). In addition, elevated calorie expenditure by HIIT may have a role as suggested by Smart et al representing that calorie burning elicits liver fat reductions and liver enzymes (31). HIIT has been shown to elicit more calorie burn compared to steady-state exercise during and following exercise training (13). Thus, it is assumed that the HIIT program can attenuate the progression of NAFLD by its potential effects on energy expenditure, glucose control, dyslipidemia, and accumulation of liver fat. This finding supports the results of Gao et al, suggesting that exercise training and dietary intervention can ameliorate fat accumulations in the liver tissue (32). Baek et al also concluded that moderate-intensity aerobic exercise training reduced fat accumulations in the liver tissue (33). It is thought that incorporating HIIT would return the elevated levels of TG and aminotransferases during continuing the high-fat diet. Although HIIT significantly ameliorated the serum levels of TG, ALT, and AST caused by the high-fat diet, they did not revert to baseline levels by exercise training. One might have speculated that a dietary intervention plays a critical role in metabolic abnormalities such as NAFLD. As suggested by Delgado et al, hepatic TG concentrations can return to baseline values by rats fed with standard chow (20).

\section{Conclusion}

In general, a high-fat diet regimen over 12 weeks significantly elevated the serum levels of TG, ALT, and AST in male Wistar rats which is an indicator of NAFLD. HIIT program ameliorated the elevated levels of these variables, but it had no potential to return the variables to baseline levels. Although exercise training may attenuate the progression of NAFLD, it may not be as potent as a dietary regimen to prevent NAFLD.

\section{Conflict of Interests}

The authors declared that they have no conflict of interests.

\section{Ethical Approval}

The study procedures complied with the codes of Helsinki Declaration for animal research and were approved by the Ethics Committee of the Islamic Azad University of Tabriz (Approval No. IR.IAU.TABRIZ.REC.1399.018).
Funding/Support

The authors received no funding for the research and publication of this manuscript.

Acknowledgements

The authors declare their gratitude to all those who assisted in conducting this study.

References

1. Carrera-Bastos P, Fontes-Villalba M, O'Keefe JH, Lindeberg S, Cordain L. The western diet and lifestyle and diseases of civilization. Res Rep Clin Cardiol. 2011;2:15-35. doi: 10.2147/rrcc.s16919.

2. Lee IM, Shiroma EJ, Lobelo F, Puska P, Blair SN, Katzmarzyk PT. Effect of physical inactivity on major noncommunicable diseases worldwide: an analysis of burden of disease and life expectancy. Lancet. 2012;380(9838):219-29. doi: 10.1016/s0140-6736(12)61031-9.

3. Byrne CD, Targher G. NAFLD: a multisystem disease. J Hepatol. 2015;62(1 Suppl):S47-64. doi: 10.1016/j. jhep.2014.12.012.

4. Nakamura A, Terauchi Y. Lessons from mouse models of high-fat diet-induced NAFLD. Int J Mol Sci. 2013;14(11):21240-57. doi: 10.3390/ijms141121240.

5. Dhibi M, Brahmi F, Mnari A, Houas Z, Chargui I, Bchir L, et al. The intake of high fat diet with different trans fatty acid levels differentially induces oxidative stress and non alcoholic fatty liver disease (NAFLD) in rats. Nutr Metab (Lond). 2011;8(1):65. doi: 10.1186/1743-7075-8-65.

6. Carmiel-Haggai M, Cederbaum AI, Nieto N. A high-fat diet leads to the progression of non-alcoholic fatty liver disease in obese rats. FASEB J. 2005;19(1):136-8. doi: 10.1096/fj.042291fje.

7. Liu Q, Bengmark S, Qu S. The role of hepatic fat accumulation in pathogenesis of non-alcoholic fatty liver disease (NAFLD). Lipids Health Dis. 2010;9:42. doi: 10.1186/1476-511x-9-42.

8. Orešič M, Hyötyläinen T, Kotronen A, Gopalacharyulu P, Nygren H, Arola J, et al. Prediction of non-alcoholic fattyliver disease and liver fat content by serum molecular lipids. Diabetologia. 2013;56(10):2266-74. doi: 10.1007/s00125013-2981-2.

9. Hou XH, Zhu YX, Lu HJ, Chen HF, Li Q, Jiang S, et al. Non-alcoholic fatty liver disease's prevalence and impact on alanine aminotransferase associated with metabolic syndrome in the Chinese. J Gastroenterol Hepatol. 2011;26(4):722-30. doi: 10.1111/j.1440-1746.2010.06509.x.

10. Suzuki A, Angulo P, Lymp J, St Sauver J, Muto A, Okada T, etal. Chronological development of elevated aminotransferases in a nonalcoholic population. Hepatology. 2005;41(1):6471. doi: 10.1002/hep.20543.

11. Pescatello LS, Riebe D, Thompson PD. ACSM's Guidelines for Exercise Testing and Prescription. Lippincott Williams \& Wilkins; 2014.

12. Slentz CA, Bateman LA, Willis LH, Shields AT, Tanner CJ, Piner LW, et al. Effects of aerobic vs. resistance training on visceral and liver fat stores, liver enzymes, and insulin resistance by HOMA in overweight adults from STRRIDE AT/RT. Am J Physiol Endocrinol Metab. 2011;301(5):E10339. doi: 10.1152/ajpendo.00291.2011.

13. Falcone PH, Tai CY, Carson LR, Joy JM, Mosman MM, McCann TR, et al. Caloric expenditure of aerobic, 
resistance, or combined high-intensity interval training using a hydraulic resistance system in healthy men. J Strength Cond Res. 2015;29(3):779-85. doi: 10.1519/ jsc.0000000000000661.

14. Yoshioka M, Doucet E, St-Pierre S, Alméras N, Richard $\mathrm{D}$, Labrie A, et al. Impact of high-intensity exercise on energy expenditure, lipid oxidation and body fatness. Int J Obes Relat Metab Disord. 2001;25(3):332-9. doi: 10.1038/ sj.ijo.0801554.

15. Fisher G, Brown AW, Bohan Brown MM, Alcorn A, Noles C, Winwood L, et al. High intensity interval- vs moderate intensity- training for improving cardiometabolic health in overweight or obese males: a randomized controlled trial. PLoS One. 2015;10(10):e0138853. doi: 10.1371/journal. pone. 0138853 .

16. Hallsworth K, Thoma C, Hollingsworth KG, Cassidy S, Anstee QM, Day CP, et al. Modified high-intensity interval training reduces liver fat and improves cardiac function in non-alcoholic fatty liver disease: a randomized controlled trial. Clin Sci (Lond). 2015;129(12):1097-105. doi: 10.1042/ cs20150308.

17. Türk Y, Theel W, Kasteleyn MJ, Franssen FME, Hiemstra PS, Rudolphus A, et al. High intensity training in obesity: a meta-analysis. Obes Sci Pract. 2017;3(3):258-71. doi: 10.1002/osp4.109.

18. Wewege M, van den Berg R, Ward RE, Keech A. The effects of high-intensity interval training vs. moderate-intensity continuous training on body composition in overweight and obese adults: a systematic review and meta-analysis. Obes Rev. 2017;18(6):635-46. doi: 10.1111/obr.12532.

19. Kalaki-Jouybari F, Shanaki M, Delfan M, GorganiFirouzjae S, Khakdan S. High-intensity interval training (HIIT) alleviated NAFLD feature via miR-122 induction in liver of high-fat high-fructose diet induced diabetic rats. Arch Physiol Biochem. 2020;126(3):242-9. doi: 10.1080/13813455.2018.1510968.

20. Srinivasan K, Viswanad B, Asrat L, Kaul CL, Ramarao P. Combination of high-fat diet-fed and low-dose streptozotocin-treated rat: a model for type 2 diabetes and pharmacological screening. Pharmacol Res. 2005;52(4):31320. doi: 10.1016/j.phrs.2005.05.004.

21. Duarte JA, Carvalho F, Pearson M, Horton JD, Browning JD, Jones JG, et al. A high-fat diet suppresses de novo lipogenesis and desaturation but not elongation and triglyceride synthesis in mice. J Lipid Res. 2014;55(12):254153. doi: 10.1194/jlr.M052308.

22. Delgado TC, Pinheiro D, Caldeira M, Castro MM, Geraldes CF, López-Larrubia P, et al. Sources of hepatic triglyceride accumulation during high-fat feeding in the healthy rat. NMR Biomed. 2009;22(3):310-7. doi: 10.1002/nbm.1327.
23. Guo R, Nair S, Zhang Y, Ren J. Adiponectin deficiency rescues high-fat diet-induced hepatic injury, apoptosis and autophagy loss despite persistent steatosis. Int J Obes (Lond). 2017;41(9):1403-12. doi: 10.1038/ijo.2017.128.

24. Khan TJ, Kuerban A, Razvi SS, Mehanna MG, Khan KA, Almulaiky YQ, et al. In vivo evaluation of hypolipidemic and antioxidative effect of 'Ajwa' (Phoenix dactylifera L.) date seed-extract in high-fat diet-induced hyperlipidemic rat model. Biomed Pharmacother. 2018;107:675-80. doi: 10.1016/j.biopha.2018.07.134.

25. Murphy MH, Blair SN, Murtagh EM. Accumulated versus continuous exercise for health benefit: a review of empirical studies. Sports Med. 2009;39(1):29-43. doi: 10.2165/00007256-200939010-00003.

26. Hao L, Wang Y, Duan Y, Bu S. Effects of treadmill exercise training on liver fat accumulation and estrogen receptor alpha expression in intact and ovariectomized rats with or without estrogen replacement treatment. Eur J Appl Physiol. 2010;109(5):879-86. doi: 10.1007/s00421-010-1426-6.

27. Botezelli JD, Mora RF, Dalia RA, Moura LP, Cambri LT, Ghezzi AC, et al. Exercise counteracts fatty liver disease in rats fed on fructose-rich diet. Lipids Health Dis. 2010;9:116. doi: 10.1186/1476-511x-9-116.

28. Tsekouras YE, Magkos F, Kellas Y, Basioukas KN, Kavouras SA, Sidossis LS. High-intensity interval aerobic training reduces hepatic very low-density lipoprotein-triglyceride secretion rate in men. Am J Physiol Endocrinol Metab. 2008;295(4):E851-8. doi: 10.1152/ajpendo.90545.2008.

29. Freese EC, Levine AS, Chapman DP, Hausman DB, Cureton KJ. Effects of acute sprint interval cycling and energy replacement on postprandial lipemia. J Appl Physiol (1985). 2011;111(6):1584-9. doi: 10.1152/japplphysiol.00416.2011.

30. Perry RJ, Samuel VT, Petersen KF, Shulman GI. The role of hepatic lipids in hepatic insulin resistance and type 2 diabetes. Nature. 2014;510(7503):84-91. doi: 10.1038/ nature 13478 .

31. Smart NA, King N, McFarlane JR, Graham PL, Dieberg G. Effect of exercise training on liver function in adults who are overweight or exhibit fatty liver disease: a systematic review and meta-analysis. Br J Sports Med. 2018;52(13):834-43. doi: 10.1136/bjsports-2016-096197.

32. Gao Y, Zhang W, Zeng LQ, Bai H, Li J, Zhou J, et al. Exercise and dietary intervention ameliorate high-fat diet-induced NAFLD and liver aging by inducing lipophagy. Redox Biol. 2020;36:101635. doi: 10.1016/j.redox.2020.101635.

33. Baek KW, Gim JA, Park JJ. Regular moderate aerobic exercise improves high-fat diet-induced nonalcoholic fatty liver disease via monoacylglycerol O-acyltransferase 1 pathway suppression. J Sport Health Sci. 2020;9(5):472-8. doi: 10.1016/j.jshs.2018.09.001. 